\title{
AVALIAÇÃO ULTRA-SONOGRÁFICA DOS MÚSCULOS MASTIGATÓRIOS E DIMENSÕES FACIAIS EM CRIANÇAS COM OCLUSÃO NORMAL E MORDIDA CRUZADA POSTERIOR UNILATERAL
}

\section{Ultrasonographic evaluation of masticatory muscles and facial dimensions in children with unilateral posterior crossbite} \author{
Paula Midori Castelo
${ }^{(1)}$, Maria Beatriz Duarte Gavião
Leonardo Rigoldi Bonjardim ${ }^{(4)}$ Luciano José Pereira ${ }^{(3)}$,
}

\section{RESUMO}

Objetivo: avaliar a espessura dos músculos masseter e porção anterior do temporal por meio da ultrasonografia e as dimensões faciais de crianças com oclusão normal e mordida cruzada posterior unilateral, na dentição decídua e mista inicial. Métodos: foram selecionadas 49 crianças, de ambos os gêneros, divididas em quatro grupos: decídua-oclusão normal, decídua-mordida cruzada, mistaoclusão normal e mista-mordida cruzada. A espessura muscular foi comparada entre os lados direito e esquerdo (oclusão normal); e normal e cruzado (mordida cruzada) (teste "t" Student), e relacionada às dimensões faciais e variáveis corporais (teste de correlação de Pearson e Spearman). As dimensões faciais foram mensuradas sobre fotografias frontais padronizadas: altura facial anterior (AFA), distância bizigomática (DB) e intergoniana (DI) e razões AFA/DB e AFA/DI. Resultados: observou-se que o músculo temporal no repouso apresentou espessura maior no lado cruzado no grupo mistacruzada $(p=0,05)$. O masseter não apresentou diferença significativa entre os lados em todos os grupos. As variáveis peso e altura não apresentaram correlação com a espessura muscular; já DB e DI apresentaram correlação positiva e as razões AFA/DB e AFA/DI, correlação negativa, com a espessura do masseter em todos os grupos, indicando que crianças com faces mais longas possuem menor espessura deste músculo. Conclusão: na amostra estudada, os exames da espessura dos músculos mastigatórios e das dimensões faciais demonstraram que a influência das características funcionais sobre as estruturas craniofaciais pôde ser observada já em crianças de pouca idade.

DESCRITORES: Ultra-Sonografia; Músculo Masséter; Músculo Temporal; Maloclusão; Face

(1) Cirurgiã Dentista; Doutoranda em Fisiologia Oral pela Faculdade de Odontologia de Piracicaba - Universidade Estadual de Campinas.

(2) Cirurgiã Dentista; Professora Titular do Departamento de Odontologia Infantil da Faculdade de Odontologia de Piracicaba - Universidade Estadual de Campinas; Mestre e Doutora em Odontopediatria pela Faculdade de Odontologia da Universidade de São Paulo.

(3) Cirurgião Dentista; Pós-Doutorando em Odontopediatria pela Faculdade de Odontologia de Piracicaba - Universidade Estadual de Campinas; Mestre e Doutor em Fisiologia Oral pela Faculdade de Odontologia de Piracicaba - Universidade Estadual de Campinas.

(4) Cirurgião Dentista; Professor Adjunto da Universidade Federal de Sergipe; Mestre e Doutor em Fisiologia Oral pela Faculdade de Odontologia de Piracicaba - Universidade Estadual de Campinas.

\section{INTRODUÇÃO}

Avanços tecnológicos têm contribuído grandemente para a avaliação anatômica e funcional das estruturas bucofaciais. A ultra-sonografia (estudo anatômico), a cinesiologia (estudo do movimento), a força de mordida, a eficiência mastigatória e a eletromiografia (estudos funcionais) são exemplos de métodos de avaliação do desempenho funcional do sistema estomatognático, capazes de auxiliar o diagnóstico de suas alterações, bem como determinar fatores etiológicos de maloclusões, permitindo prognosticar e avaliar a evolução de tratamentos instituídos.

Diversos estudos in vivo demonstraram a viabilidade e a aplicabilidade da ultra-sonografia no estudo de músculos superficiais, como os músculos 
mastigatórios, por ser um método não invasivo, de fácil manuseio e baixo custo. Além disso, não apresenta dificuldades em sua realização, como a ressonância magnética, que requer sedação em crianças menores de dez anos de idade, e nem os efeitos biológicos cumulativos de outros exames de imagem, como a tomografia computadorizada ${ }^{1-7}$. Esta técnica permite acesso fácil e reprodutível da espessura muscular, obtendo-se a informação quantitativa da sua capacidade funcional, além de propiciar a determinação de alterações musculares estruturais ${ }^{8,9}$.

A mordida cruzada é uma relação bucal, labial ou lingual anormal entre dentes superiores e inferiores, quando em oclusão. Pode incluir um ou mais dentes de cada arco, ser funcional ou esquelética, além de estar presente uni ou bilateralmente ${ }^{10}$. Muitos estudos buscaram avaliar suas prováveis causas, sejam de origem genética ou de desenvolvimento; sendo, esta última, apoiada em três hipóteses principais: hábitos de sucção, obstrução das vias respiratórias e mudanças nos hábitos alimentares, com conseqüente alteração da função mastigatória ${ }^{11,12}$.

A epidemiologia e conseqüências da presença de maloclusões em crianças têm constituído objeto de pesquisa para se determinar qual a época correta para prevenção e intervenção dessas alterações. Estudos demonstraram que este tipo de maloclusão está associado às alterações da função mastigatória em crianças, como, por exemplo, assimetria da atividade dos músculos mastigatórios ${ }^{13-15}$ e menor eficiência mastigatória ${ }^{16}$; além disso, muitos autores afirmam ser um tipo de maloclusão que freqüentemente não apresenta auto-correção ${ }^{10,17}$.

A mastigação, sendo uma atividade neuromuscular aprendida e adaptada durante toda a vida, desenvolve-se concomitantemente com o crescimento craniofacial, com o aumento do volume intrabucal, com os dentes em irrupção e com a maturação dos músculos e articulações, sob coordenação e interação das funções realizadas pelo sistema nervoso central. Daí a importância de que o estado de normalidade neuromuscular, esquelético e das estruturas dentais seja atingido já na dentição decídua ${ }^{10,18-20}$.

Dada a efetividade dos métodos de avaliação mencionados, torna-se importante utilizá-los de modo a se obter parâmetros comparativos entre os diversos tipos de características morfológicas e funcionais nesta fase, podendo-se acompanhar objetivamente os processos de crescimento e desenvolvimento, diagnosticando precocemente alterações que possam se perpetuar. Além disso, são raros os estudos realizados a respeito das características estruturais e funcionais associadas à mordida cruzada posterior em crianças de pouca idade.

Sendo assim, este estudo buscou avaliar a espessura dos músculos masseter e porção anterior do músculo temporal em crianças na fase da dentição decídua e dentição mista inicial com oclusão normal e mordida cruzada posterior unilateral, correlacionando com as dimensões faciais e com as variáveis corporais.

\section{MÉTODOS}

Foram examinadas crianças de ambos os gêneros, entre três anos e meio e sete anos, cadastradas para tratamento no Departamento de Odontologia Infantil - Faculdade de Odontologia de Piracicaba Unicamp Universidade Estadual de Campinas. A amostra consistiu de 49 crianças, que foram selecionadas após o consentimento verbal e escrito dos responsáveis para participarem do estudo. A seleção foi realizada após completa anamnese e exame clínico, nos quais verificou-se o bom estado de saúde geral, a normalidade de estruturas orofaciais, o peso e a altura corporais, a oclusão morfológica e o estágio da dentição: dentição decídua completa ou dentição mista - fase inicial (primeiros molares e incisivos em irrupção) ${ }^{18,21}$.

Sujeitos portadores de anomalias dentárias de forma, estrutura ou número, lesões de cárie e/ou mordida cruzada bilateral foram excluídos.

Assim, as crianças foram divididas em quatro grupos de acordo com o estágio e o tipo de oclusão (normal ou mordida cruzada posterior unilateral funcional): grupo DN (decídua-normal, $n=15)$, grupo DC (decídua-cruzada, $\mathrm{n}=10$ ), grupo $\mathrm{MN}$ (mista-normal, $n=13$ ), grupo MC (mista-cruzada, $n=11$ ). As crianças com oclusão normal não apresentavam sinais e/ou sintomas de disfunção temporomandibular ${ }^{22}$, nem desvio de linha média.

Para os exames de ultra-sonografia, os lados dos arcos dentários foram divididos em esquerdo e direito (oclusão normal), e normal e cruzado (mordida cruzada posterior unilateral).

\section{Espessura dos Músculos Mastigatórios}

A espessura dos músculos mastigatórios foi avaliada por meio de ultra-sonografia (Just Vision Toshiba $^{\circledR}$, Japão, $56 \mathrm{~mm} / 10 \mathrm{MHz}$ transdutor linear, do Departamento de Odontologia Infantil, Área de Odontopediatria - FOP - UNICAMP). As aquisições de imagens foram realizadas para os músculos masseter e porção anterior do músculo temporal, de ambos os lados, nas posições mandibulares de repouso e de máxima intercuspidação.

O transdutor foi posicionado transversalmente em relação à direção das fibras musculares, considerando-se que o ventre do músculo masseter localiza-se, aproximadamente, dois centímetros $(\mathrm{cm})$ acima do ângulo da mandíbula em direção à pálpebra superior e à porção anterior do músculo temporal, e cerca 1,5 
cm para trás e para cima da comissura palpebral externa. Estas localizações foram confirmadas através de palpação e movimentação do transdutor para se obter uma imagem otimizada. Posicionando-se obliquamente o transdutor, a espessura muscular torna-se aumentada; portanto, a angulação utilizada para se evitar esta alteração deve buscar a melhor definição da imagem do ramo mandibular e do osso temporal.

Durante o exame, as crianças permaneceram sentadas, recostadas, sem fixação da cabeça. As medições foram realizadas diretamente sobre a imagem obtida na tela do aparelho, no momento da sua aquisição, com aproximação de $0,1 \mathrm{~mm}$. Foram realizados três exames em cada músculo, para cada posição mandibular, com um intervalo de dois minutos entre cada mensuração (Figuras 1, 2, 3 e 4).

\section{Análise das Dimensões Faciais}

Fotografias frontais de cada criança foram obtidas por meio de metodologia padronizada, utilizando-se câmera fotográfica analógica com flash automático (Canon AE-1), montada em tripé e filme negativo. Os sujeitos permaneceram em pé à frente de um fundo claro, sob luz natural e posição relaxada. A cabeça foi posicionada com o plano sagital e o plano de Frankfo(u)rt, respectivamente, perpendicular e paralelo ao solo, dentes em máxima intercuspidação ${ }^{1,9}$ e lábios em repouso. A altura da câmera foi determinada de forma que a face da criança ficasse centralizada nos planos vertical e horizontal. A distância entre a câmera fotográfica e o fundo foi padronizada em 105 $\mathrm{cm}$ para todos os sujeitos. Duas fotografias (10)15 $\mathrm{cm}$, coloridas) foram obtidas de cada criança e a meIhor foi escolhida para a obtenção das medidas.

As mensurações foram realizadas sobre as fotografias utilizando-se papel ultrafan, lápis, esquadro, transferidor e paquímetro digital (Digimatic, série 500, Mitutoyo). Os planos e pontos antropométricos considerados foram ${ }^{1,3,5,9,23,24}$.

- plano mandibular (plano que tangencia a distância entre o ângulo da mandíbula e o mento, no perfil mole);

- plano do ramo mandibular (plano que tangencia a distância entre o ângulo da mandíbula e o arco zigomático, no perfil mole);

- ponto pupilar (localizado no centro da pupila) e plano pupilar (plano que passa entre as duas pupilas com o sujeito olhando para o infinito);

- zygion (ponto mais lateral do arco zigomático);

- gonion (ponto determinado pela bissetriz do ângulo formado pelo plano mandibular e plano do ramo mandibular).

As grandezas consideradas para a análise das dimensões faciais estão na Tabela 1, sendo que as razões AFA/DB (altura facial anterior/distância bizigomática) e AFA/DI (altura facial anterior/distância intergoniana) também foram avaliadas. Todas as medidas foram determinadas com precisão de $0,01 \mathrm{~mm}$ e a média de duas mensurações foi considerada como valor final ${ }^{3}$.

O presente estudo foi aprovado pelo Comitê de Ética em Pesquisa da Faculdade de Odontologia de Piracicaba - UNICAMP sob protocolos n. 147/2001 e 148/2002.

\section{Análise Estatística}

O erro de medida do exame ultra-sonográfico foi calculado pela fórmula de Dahlberg's: ${ }^{1,3} S_{e}=\sqrt{ } \sum(m 1$ - m2) ${ }^{2} / 2 n$; em que: $S_{\mathrm{e}}=$ erro de medida; $m 1=$ primeira medição; $m 2$ = segunda medição; $n$ = número de sujeitos. A segunda medição foi realizada uma semana após a primeira.

Os resultados foram tabulados e aplicou-se a análise estatística descritiva para as variáveis estudadas, que consistiram de porcentagem, média e desvio padrão; sendo que cada fase da dentição (decídua/ mista inicial) foi avaliada separadamente. A espessura dos músculos masseter e porção anterior do temporal foi comparada entre o lado esquerdo e direito, e lados cruzado e normal, nos grupos com oclusão normal e mordida cruzada, respectivamente, pelo teste " $\mathrm{t}$ " de Student pareado. A espessura muscular entre os grupos com normoclusão e maloclusão, dentro de cada fase da dentição, foi comparada pela Análise de Variância (Anova), considerando-se os lados direito e esquerdo, cruzado e normal, respectivamente. A espessura muscular e as dimensões faciais foram correlacionadas pela aplicação do teste de correlação de Pearson e Spearman.

\section{RESULTADOS}

Os valores da média e desvio padrão da espessura dos músculos masseter e porção anterior do temporal, em repouso e em máxima intercuspidação, das dimensões faciais e das variáveis corporais, para os quatro grupos, estão apresentados na Tabela 2.

Os resultados obtidos mostraram que nos grupos de dentição decídua normal (DN) e com mordida cruzada (DC) não houve diferença significativa na espessura muscular entre os lados, tanto em máxima intercuspidação, como no repouso. Na dentição mista inicial, tanto na oclusão normal (MN) como na mordida cruzada (MC), não houve diferença na espessura do músculo masseter entre os lados, em ambas as posições mandibulares. Já no grupo MC, a porção anterior do temporal do lado cruzado apresentou espessura estatística e significativamente maior em relação ao lado normal no repouso $(p \leq 0,05)$. Pela Análise de Variância (Anova) constatou-se não haver diferença significativa na espessura muscular 
entre os grupos com normoclusão e maloclusão nas respectivas fases das dentições avaliadas. Os resultados do cálculo do erro de medida do exame ultrasonográfico são mostrados na Tabela 3.

Para o cálculo dos Coeficientes de Correlação (Teste de Correlação de Pearson) entre a espessura muscular, as variáveis corporais e as dimensões faciais foram considerados os valores das médias da espessura muscular entre lados para os músculos masseter (nos grupos DN, DC, MN e MC) e temporal (nos grupos DC, DN e MN), enquanto somente o músculo temporal no repouso do grupo $\mathrm{MC}$ teve seus lados analisados separadamente pelo Teste de Correlação de Spearman, uma vez que somente neste caso houve diferença significativa na espessura entre os lados cruzado e normal. Os Coeficientes de Correlação obtidos estão presentes na Tabela 4.
No grupo DC houve correlação positiva significativa somente entre altura e espessura do masseter no repouso; nos grupos de dentição mista, a espessura muscular não apresentou correlação significativa com as variáveis corporais. O grupo DN não apresentou correlação estatisticamente significativa entre espessura muscular e dimensões faciais. A variável AFA apresentou correlação significativa com o músculo temporal anterior no repouso do grupo MC; já as variáveis DB e DI obtiveram coeficientes estatisticamente significativos e positivos, ou próximos do nível de significância com a espessura do músculo masseter. Os coeficientes de correlação obtidos entre as razões AFA/DB e AFA/DI e a espessura do masseter foram negativos e, alguns, estatisticamente significativos. A porção anterior do músculo temporal não mostrou relacionar-se às variáveis DB e DI.

Tabela 1 - Grandezas consideradas para a análise das dimensões faciais

\begin{tabular}{ccc}
\hline \multicolumn{1}{c}{ Variámbolo } & Definição \\
\hline Altura facial anterior & AFA & Distância entre a linha interpupilar e o mento \\
Distância bizigomática & DB & Distância entre o zygion esquerdo e o direito \\
Distância intergoniana & DI & Distância entre o gonion esquerdo e o direito \\
\hline
\end{tabular}

Tabela 2 - Distribuição da amostra e valores da média (†DP) para espessura dos músculos masseter e porção anterior do temporal $(\mathrm{mm})$, dimensões faciais $(\mathrm{mm})$, peso $(\mathrm{Kg})$ e altura $(\mathrm{m})$

\begin{tabular}{|c|c|c|c|c|c|c|c|c|c|}
\hline \multicolumn{2}{|l|}{ Grupo } & \multicolumn{2}{|c|}{ DN } & \multicolumn{2}{|c|}{ DC } & \multicolumn{2}{|c|}{ MN } & \multicolumn{2}{|c|}{ MC } \\
\hline \multicolumn{2}{|l|}{$\mathrm{n}$} & \multicolumn{2}{|c|}{15} & \multicolumn{2}{|c|}{10} & \multicolumn{2}{|c|}{13} & \multicolumn{2}{|c|}{11} \\
\hline Lado & & Esquerdo & Direito & Normal & Cruzado & Esquerdo & Direito & Normal & Cruzado \\
\hline \multirow{4}{*}{ Masseter } & \multirow{2}{*}{ RE } & 9,36 & 9,38 & 9,78 & 9,76 & 10,54 & 10,37 & 10,13 & 9,88 \\
\hline & & $\pm 1,06$ & $\pm 0,77$ & $\pm 0,94$ & $\pm 0,79$ & $\pm 0,98$ & $\pm 0,97$ & $\pm 1,16$ & $\pm 1,25$ \\
\hline & \multirow{2}{*}{ MI } & 10,92 & 11,15 & 11,43 & 11,25 & 12,16 & 12,17 & 11,88 & 11,67 \\
\hline & & $\pm 1,06$ & $\pm 0,95$ & $\pm 1,40$ & $\pm 1,13$ & $\pm 1,29$ & $\pm 1,21$ & $\pm 1,33$ & $\pm 1,35$ \\
\hline \multirow{4}{*}{ Temporal } & \multirow{2}{*}{ RE } & 2,59 & 2,54 & 2,59 & 2,65 & 2,76 & 2,72 & $2,63^{\mathrm{a}}$ & $2,68^{b}$ \\
\hline & & $\pm 0,14$ & $\pm 0,18$ & $\pm 0,19$ & $\pm 0,21$ & $\pm 0,26$ & $\pm 0,23$ & $\pm 0,21$ & $\pm 0,18$ \\
\hline & \multirow{2}{*}{ MI } & 3,52 & 3,42 & 3,24 & 3,38 & 3,52 & 3,49 & 3,44 & 3,46 \\
\hline & & $\pm 0,26$ & $\pm 0,30$ & $\pm 0,23$ & $\pm 0,26$ & $\pm 0,33$ & $\pm 0,30$ & $\pm 0,39$ & $\pm 0,31$ \\
\hline AFA & & \multicolumn{2}{|c|}{$52,74 \pm 2,43$} & \multicolumn{2}{|c|}{$53,20 \pm 1,94$} & \multicolumn{2}{|c|}{$55,63 \pm 2,56$} & \multicolumn{2}{|c|}{$55,10 \pm 2,96$} \\
\hline DB & & \multicolumn{2}{|c|}{$69,43 \pm 3,11$} & \multicolumn{2}{|c|}{$70,52 \pm 3,16$} & \multicolumn{2}{|c|}{$73,67 \pm 2,94$} & \multicolumn{2}{|c|}{$70,96 \pm 4,96$} \\
\hline
\end{tabular}




$\begin{array}{ccccc}\text { DI } & 53,62 \pm 3,22 & 53,68 \pm 3,78 & 57,04 \pm 3,46 & 56,23 \pm 4,22 \\ \text { AFA/DB } & 0,76 \pm 0,03 & 0,76 \pm 0,02 & 0,76 \pm 0,03 & 0,78 \pm 0,04 \\ \text { AFA/DI } & 0,99 \pm 0,05 & 0,99 \pm 0,06 & 1,00 \pm 0,05 & 0,98 \pm 0,04 \\ \text { Peso } & 20,12 \pm 4,20 & 20,39 \pm 4,04 & 25,95 \pm 4,44 & 23,72 \pm 7,00 \\ \text { Altura } & 1,09 \pm 0,07 & 1,10 \pm 0,07 & 1,18 \pm 0,05 & 1,17 \pm 0,06\end{array}$

DN, decídua-normal; DC, decídua-cruzada; MN, mista-normal; MC, mista-cruzada; RE, repouso; MI, máxima intercuspidação; AFA, altura facial anterior; DB, distância bizigomática; DI, distância intergoniana; DP, desvio padrão; mm, milímetro; Kg, quilograma; $m$, metro

$a \neq b(p \leq 0,05 ;$ teste "t" Student - pareado)

Tabela 3 - Erro de medida (Se) da espessura dos músculos masseter e porção anterior do temporal avaliado em 15 sujeitos

\begin{tabular}{lcc}
\hline & Se $(\mathrm{mm})$ & $\%$ erro \\
\hline Masseter esquerdo / repouso & 0,53 & 5,78 \\
Masseter esquerdo / máxima intercuspidação & 0,36 & 3,18 \\
Temporal esquerdo / repouso & 0,09 & 3,63 \\
Temporal esquerdo / máxima intercuspidação & 0,15 & 4,38 \\
$\begin{array}{l}\mathrm{Se}=\sqrt{\Sigma}(\mathrm{m} 1-\mathrm{m} 2)^{2} / 2 \mathrm{n} \\
\%=(\mathrm{Se} / \mathrm{média}) \times 100\end{array}$ & &
\end{tabular}

Tabela 4 - Coeficientes de correlação ( $r$ ) entre a espessura dos músculos masseter e porção anterior do temporal, dimensões faciais e as variáveis corporais peso e altura

\begin{tabular}{|c|c|c|c|c|c|c|c|c|c|c|c|c|c|c|c|c|c|}
\hline \multirow[t]{3}{*}{ Grupo } & \multicolumn{4}{|c|}{ DN } & \multicolumn{4}{|c|}{ DC } & \multicolumn{4}{|c|}{ MN } & \multicolumn{5}{|c|}{ MC } \\
\hline & \multicolumn{2}{|c|}{ Masseter } & \multicolumn{2}{|c|}{ Temporal } & \multicolumn{2}{|c|}{ Masseter } & \multicolumn{2}{|c|}{ Temporal } & \multicolumn{2}{|c|}{ Masseter } & \multicolumn{2}{|c|}{ Temporal } & \multicolumn{2}{|c|}{ Masseter } & \multirow[t]{2}{*}{ Temporal } & \multicolumn{2}{|c|}{$\begin{array}{c}\text { Temporal } \\
\text { RE } \\
\end{array}$} \\
\hline & RE & MI & RE & $\mathrm{MI}$ & RE & MI & RE & $\mathrm{MI}$ & RE & MI & RE & MI & RE & $\mathrm{MI}$ & & $\begin{array}{c}\text { Lado } \\
\text { normal }\end{array}$ & $\begin{array}{c}\text { Lado } \\
\text { cruzado }\end{array}$ \\
\hline AFA & 0,20 & 0,24 & $-0,09$ & 0,15 & 0,48 & $-0,22$ & 0,14 & 0,18 & 0,04 & 0,02 & $-0,17$ & $-0,21$ & 0,30 & 0,27 & 0,46 & $0,67^{a}$ & $0,61^{a}$ \\
\hline DB & 0,37 & 0,42 & 0,00 & 0,32 & $0,65^{*}$ & 0,54 & 0,05 & 0,30 & $0,57^{*}$ & 0,44 & 0,29 & 0,14 & 0,59 & 0,53 & 0,30 & 0,17 & 0,17 \\
\hline DI & 0,35 & 0,28 & 0,20 & 0,37 & 0,62 & 0,62 & $-0,26$ & 0,04 & 0,27 & 0,22 & $-0,16$ & $-0,24$ & $0,61^{*}$ & $0,60^{*}$ & 0,44 & 0,46 & 0,42 \\
\hline AFA/DB & $-0,24$ & $-0,26$ & $-0,07$ & $-0,20$ & $-0,51$ & $-0,67^{*}$ & 0,02 & $-0,36$ & $-0,56$ & $-0,48$ & $-0,52$ & $-0,39$ & $-0,46$ & $-0,41$ & 0,06 & 0,36 & 0,36 \\
\hline AFA/DI & $-0,18$ & $-0,08$ & $-0,25$ & $-0,25$ & $-0,46$ & $-0,62$ & 0,40 & 0,06 & $-0,27$ & $-0,23$ & 0,10 & 0,15 & $-0,65^{\star}$ & $-0,67^{*}$ & $-0,15$ & $-0,03$ & 0,02 \\
\hline Peso & 0,27 & 0,23 & 0,18 & 0,40 & 0,54 & 0,40 & 0,07 & 0,22 & 0,09 & 0,15 & $-0,18$ & $-0,16$ & 0,35 & 0,35 & 0,37 & 0,46 & 0,45 \\
\hline Altura & 0,15 & 0,22 & 0,16 & 0,49 & $0,78^{*}$ & 0,63 & 0,02 & 0,43 & 0,00 & 0,05 & $-0,33$ & $-0,39$ & 0,22 & 0,25 & 0,32 & 0,53 & 0,48 \\
\hline
\end{tabular}

${ }^{*} \mathrm{p} \leq 0,05$ (teste de correlação de Pearson)

$a=p \leq 0,05$ (teste de correlação de Spearman)

Abreviações: DN, decídua-normal; DC, decídua-cruzada; MN, mista-normal; MC, mista-cruzada, RE, repouso; MI, máxima intercuspidação; AFA, altura facial anterior; DB, distância bizigomática; DI, distância intergoniana 


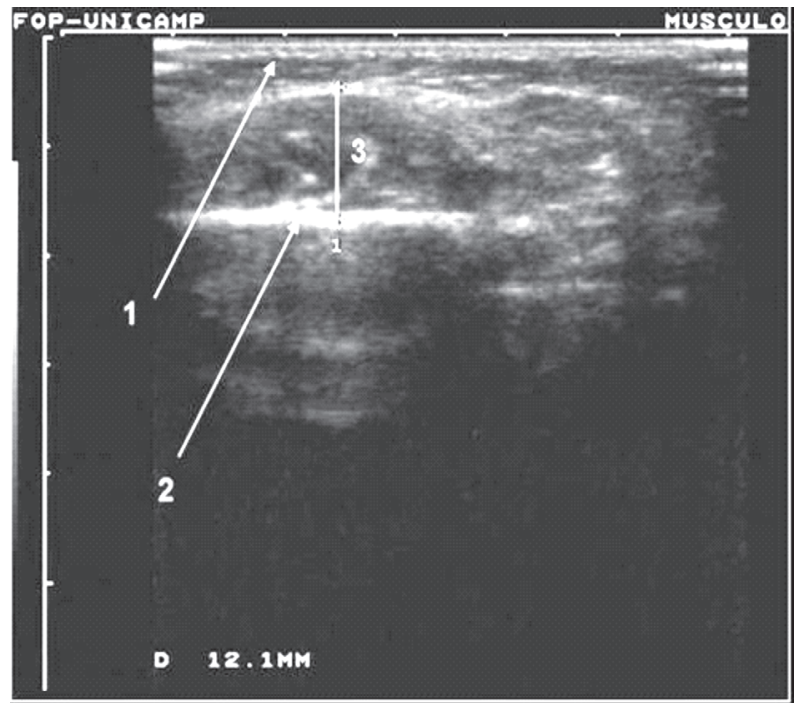

Figura 1 - Imagem ultra-sonográfica do músculo masseter em repouso: superfície do transdutor (1), ramo da mandíbula (2) e espessura do músculo masseter (3)

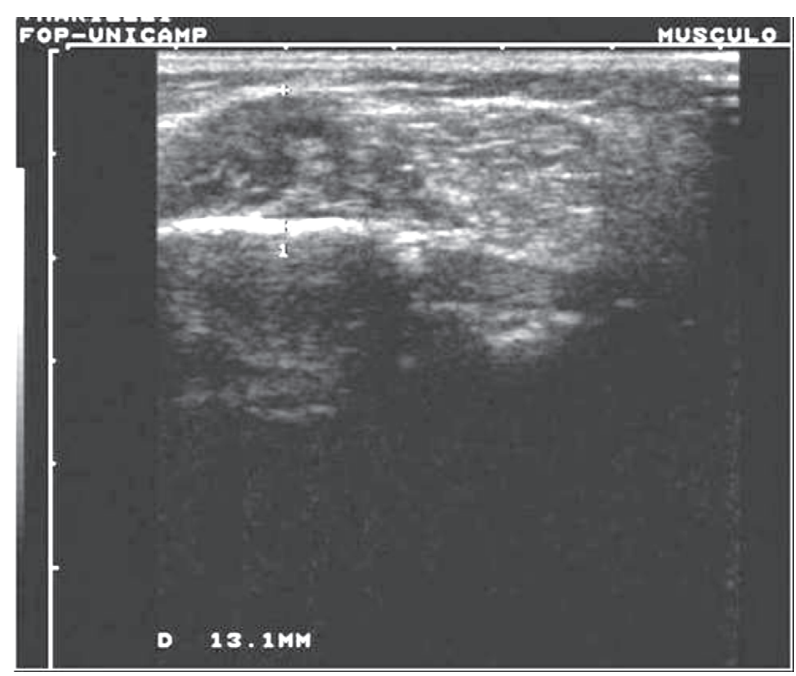

Figura 2 - Imagem ultra-sonográfica do músculo masseter durante a máxima intercuspidação

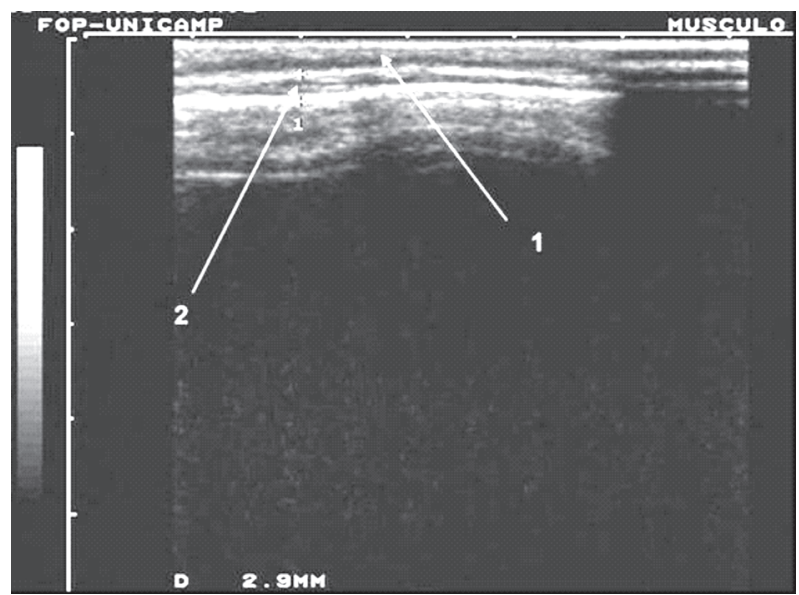

Figura 3 - Imagem ultra-sonográfica da porção anterior do músculo temporal em repouso: superfície do transdutor (1) e região mais espessa do músculo (2)

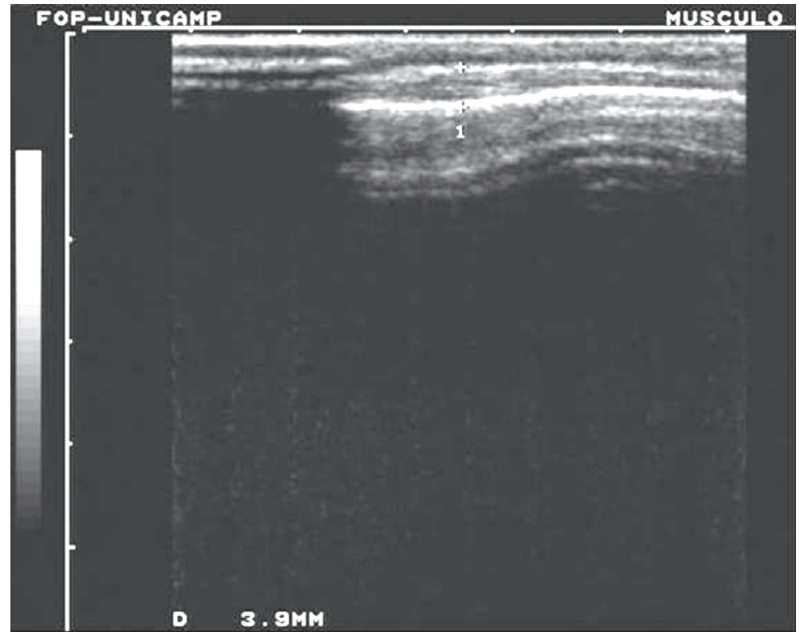

Figura 4 - Imagem ultra-sonográfica da porção anterior do músculo temporal durante a máxima intercuspidação

\section{DISCUSSÃO}

A qualidade da função mastigatória é dependente de uma série de fatores: área oclusal, número de dentes, atividade, dimensões e coordenação dos músculos mastigatórios, dimensões craniofaciais e ação da língua e dos músculos peri-bucais na manipulação do alimento ${ }^{25}$. No estudo das funções que abrangem o sistema estomatognático, sobretudo em crianças, é fundamental considerar a idade, o estágio do desenvolvimento dentário e o tipo de oclusão como critérios de seleção da amostra, para evitar variações nos resultados ${ }^{18,26,27}$.

Pela ultra-sonografia foi possível obter imagens bem definidas dos músculos masseter e porção anterior do temporal e determinar sua espessura com grande reprodutibilidade, rapidez e sem que a criança fosse exposta à radiação, o que torna esta técnica adequada para a avaliação dos músculos peribucais. Comparando os valores absolutos das mensurações musculares em diferentes estudos, observam-se discrepâncias que são devidas às diferenças no tipo de amostra, na localização do transdutor para a captura de imagens e no aparelho e técnica utilizados ${ }^{4}$. No presente estudo, a posição do transdutor para a realização do exame foi determinada por palpação dos músculos, em repouso e em contração ${ }^{7}$. Seu correto posicionamento foi confirmado observando a imagem na tela do aparelho de ultra-som, sendo que as imagens da superfície do ramo mandibular, relativo ao músculo masseter, e do osso temporal, relativo à porção anterior do temporal, deveriam estar bem nítidas. Caso o transdutor fosse posicionado obliquamente, a imagem do músculo ficaria aumentada ${ }^{1}$.

É importante considerar, também, que quanto maior a freqüência do transdutor, mais nítidas e claras serão as imagens capturadas ${ }^{28}$. Utilizou-se neste 
estudo um transdutor de $10 \mathrm{MHz}$, superior aos transdutores utilizados em estudos anteriores, que variaram de 5,0 a 7,5 MHz. Conseqüentemente, o erro de medida pôde ser considerado baixo (Tabela 3), sendo que para o músculo masseter em repouso este valor foi superior ao da máxima intercuspidação (0,53 mm e 0,36 mm, respectivamente), valores estes similares a outros estudos ${ }^{1,3}$. O erro na contração muscular foi inferior aos dos referidos estudos $(0,49 \mathrm{~mm}$ e $0,45 \mathrm{~mm}$, respectivamente). Esta diferença de valores entre as duas posições mandibulares se deve à maior vulnerabilidade do músculo em repouso, às variações de pressão que o transdutor exerce sobre a bochecha, sendo o masseter conseqüentemente mais susceptível a esta pressão, o que determinou maior erro de medida para o masseter em relação ao temporal. Observou-se, portanto, em todas as mensurações realizadas, que o músculo contraído apresentou espessura maior que a do músculo relaxado, corroborando os resultados anteriores ${ }^{1,3,6-8,28,29}$.

Os valores da espessura do músculo masseter foram superiores aos da porção anterior do temporal, em todos os grupos. Outros autores encontraram resultados semelhantes, em crianças com dentição mista ${ }^{29}$, enquanto outro grupo relatou ter encontrado valores altos para a espessura do músculo temporal no repouso (média de $14,35 \mathrm{~mm}$ em adultos) ${ }^{5}$, superiores até mesmo aos valores do músculo masseter do mesmo estudo. Esta diferença deve-se, provavelmente, às variações no posicionamento do transdutor e à porção do músculo que foi avaliada.

No presente estudo, os grupos DN e DC e o grupo MN não apresentaram diferenças significativas na espessura muscular entre os lados (Tabela 2). No grupo MC a porção anterior do músculo temporal em repouso do lado cruzado apresentou-se significativamente mais espesso em relação ao lado normal $(p \leq 0,05)$.

A menor espessura encontrada no lado normal poderia ser devida à menor solicitação muscular para a manutenção do côndilo dentro da cavidade glenóide. Isto porque o côndilo do lado cruzado apresenta-se posicionado mais superior e posteriormente na cavidade do que o côndilo do lado normal ${ }^{30}$. Além disso, crianças com mordida cruzada posterior unilateral podem apresentar maior atividade do músculo temporal posterior no lado cruzado no repouso e em máxima intercuspidação ${ }^{13}$ e posição postural assimétrica, isto é, a mandíbula no repouso ainda permanece deslocada para o lado cruzado ${ }^{31}$.

Em relação ao músculo masseter, os resultados não mostraram diferenças significativas em ambas as fases das dentições. No entanto, outro estudo ${ }^{6}$ apontou que o masseter no lado cruzado apresentou espessura estatisticamente menor do que o lado normal, em crianças na fase de dentição mista; após três anos da correção da mordida cruzada, esta dife- rença já não foi significativa, sugerindo que o tratamento eliminou a assimetria muscular. De acordo com a teoria de Planas ${ }^{32}$ o sujeito portador de mordida cruzada posterior unilateral, mastiga preferentemente no lado cruzado, e como conseqüência, o masseter do lado cruzado deveria apresentar-se hipertrofiado pela maior demanda funcional ${ }^{8,33}$. No entanto, estudos anteriores também demonstraram que não há predileção pelo lado cruzado durante a mastigação em sujeitos portadores de mordida cruzada ${ }^{34,35}$.

Apesar da diferença na faixa etária da amostra, os resultados encontrados no presente estudo concordam com resultados de estudo anterior ${ }^{3}$, que avaliou sujeitos entre sete e 22 anos de idade com diferentes tipos de oclusão, observando que a espessura do músculo masseter entre os lados esquerdo e direito não diferiu significativamente. Também houve concordância com os resultados de estudo, no qual crianças na fase de dentição mista, com mordida aberta e sobremordida, apresentaram maior espessura do músculo temporal anterior em relação àquelas portadoras de oclusão normal, enquanto o músculo masseter não apresentou tal diferença ${ }^{29}$.

A comparação entre os grupos com normoclusão e maloclusão, entre os respectivos grupos e dentro de cada fase da dentição considerada, mostrou não haver diferença significativa na espessura dos músculos, em ambas as posições mandibulares. Pode-se sugerir que a faixa etária e as fases das dentições apresentadas pela amostra selecionada poderiam ser de influência.

Na dentição decídua, o aspecto funcional da mordida cruzada ${ }^{10}$ poderia não estar determinando alterações morfológicas, sendo que as assimetrias funcionais e posturais oriundas do cruzamento ainda não estariam influenciando a estrutura muscular dos músculos avaliados. Na fase da dentição mista, espera-se que algumas alterações dento-alveolares, decorrentes da mordida cruzada, já estejam evidenciando-se. No entanto, de acordo com os resultados do presente estudo, observa-se que a maloclusão ainda não determinou diferenças significativas na espessura do músculo masseter, quando foram comparados os grupos na fase de dentição mista. Isto também pode ser explicado pela fase da dentição mista avaliada, isto é, a fase inicial, na qual alterações dimensionais ainda não puderam ser detectadas. As dimensões musculares são preditoras da quantidade de força que um músculo é capaz de gerar ${ }^{5,36}$; assim, as alterações funcionais decorrentes da presença de maloclusão podem ser observadas pelo decréscimo na máxima força de mordida em pré-adolescentes e adultos ${ }^{18}$, mas não em crianças em idade pré-escolar ${ }^{19,37}$.

Ao avaliar a influência das variáveis corporais sobre a espessura muscular, observou-se que somente a espessura do masseter no repouso do grupo DC apresentou correlação positiva e significativa com a altura corporal. Não houve correlação significativa entre as variá- 
veis nos grupos de dentição mista (Tabela 4). Observando os coeficientes obtidos, conclui-se que outros fatores devem atuar como preditores do aumento ou da diminuição da espessura muscular. Comparações com os resultados obtidos em crianças na fase de dentição decídua não foram possíveis, por não terem sido encontrados dados publicados até o momento. Meninas e meninos apresentam massa muscular semelhante, sendo que as diferenças tornam-se significativas na puberdade, sob influência dos hormônios sexuais ${ }^{38}$. Em indivíduos jovens, o músculo masseter apresentou correlação significativa e positiva com o peso e a altura ${ }^{3}$, e somente com o peso, em indivíduos adultos ${ }^{1,5}$. No presente estudo, o peso corporal não teve influência na espessura muscular, sugerindo que crianças mais pesadas não necessariamente apresentam músculos mais espessos, mas massa adiposa maior.

Através da utilização de fotografias frontais padronizadas na análise das dimensões faciais, evita-se que a criança seja exposta à radiação, como ocorre nos estudos cefalométricos. Este tipo de estudo já obteve resultados importantes na avaliação da influência do padrão facial sobre as funções que envolvem o sistema estomatognático ${ }^{1,9,39}$. Outro método utilizado para a análise facial é a antropometria, no qual as mensurações são realizadas diretamente sobre a face do sujeito ${ }^{3,5,40}$. Observou-se no presente estudo que até a fase inicial da dentição mista, a diferença na forma facial entre os gêneros não foi significativa, demonstrado pelo baixo desvio padrão das dimensões faciais (Tabela 2), corroborando estudos anteriores ${ }^{9,26,41}$. Já em adultos, a variação da morfologia facial é grande e difere de indivíduo para indivíduo ${ }^{9,42}$.

O conhecimento das influências funcionais e genéticas sobre o crescimento e desenvolvimento craniofacial é ainda limitado e difícil de quantificar ${ }^{43}$. Observa-se que indivíduos portadores de padrão braquicefálico são caracterizados por menor altura facial anterior, menor inclinação da mandíbula, ângulo goníaco menos obtuso e paralelismo entre os planos mandibular e palatino; aqueles com padrão dolicocefálico apresentam maior altura facial anterior, maior inclinação do plano mandibular e ângulo goníaco mais obtuso ${ }^{9,44}$. Estudos anteriores avaliaram a função dos músculos mastigatórios através da eletromiografia ${ }^{8,41}$, força de mordida ${ }^{5,9,26,27,38,42,45}$, da determinação de suas dimensões ${ }^{1,3,4,8,28}$ e sua relação com a morfologia craniofacial em adultos e indivíduos em crescimento. Assim como o posicionamento vertical dos músculos elevadores pode direcionar o crescimento mandibular e determinar um ângulo goníaco menos obtuso e sobremordida ${ }^{46}$, o tipo de fibra que compõe o músculo masseter pode ser de importância ${ }^{43}$. Além disso, foi observado que crianças com padrão dolicocefálico possuem maior altura das coroas clínicas dos dentes superiores e inferiores, além de aumento no componente dento-alveolar, provavelmente devido à menor força de mordida que elas apresentam ${ }^{27,41}$ e que permite sobre-irrupção dos elementos dentários ${ }^{44}$.

No presente estudo, observou-se fraca correlação entre a espessura muscular e a altura facial anterior (AFA), de acordo com dados de estudo anterior ${ }^{28}$. Isto poderia ser devido às variações mais significativas da morfologia craniofacial concentrar-se na região inferior da face, abaixo do plano palatino ${ }^{44}$. A variável DI (distância intergoniana) apresentou coeficientes de correlação positivos e, alguns, significativos com a espessura do músculo masseter; um dos motivos deste achado é, justamente, pela variável DI incluir a espessura deste músculo ${ }^{1}$. A distância bizigomática (DB) apresentou coeficientes positivos e significativos, ou bastante próximos do nível de significância, com a espessura do masseter, sugerindo que a maior dimensão transversal da face, que a torna mais larga, está relacionada à maior espessura muscular (Tabela 4). As razões AFA/DB e AFA/DI apresentaram coeficientes negativos ou valores próximos a zero, mas não significativos em sua maioria com a espessura muscular; reforçando a tendência de crianças com faces mais longas de apresentar menor espessura do masseter. Este tipo de relação mostrou-se estatisticamente significativa em mulheres ${ }^{1}$, onde a espessura do masseter correlacionou-se negativamente com as razões $\mathrm{AFA} / \mathrm{DB}$ e AFA/DI, obtidas também por meio de fotografias frontais.

Utilizando a antropometria em indivíduos em crescimento, foram encontradas correlações estatisticamente significativas e positivas entre a espessura do masseter e as distâncias intergoniana e bizigomática; e correlação negativa com a altura facial anterior inferior (subnasal-mento) ${ }^{3}$. De forma semelhante, concluíram que indivíduos que possuíam menor espessura do masseter apresentavam a face mais longa. Existem muitos trabalhos que buscaram esta relação através da cefalometria e ultra-sonografia em adultos, determinando variáveis craniofaciais lineares e angulares sobre telerradiografias. Observou-se que a maior espessura do músculo masseter está relacionada à maior altura do ramo mandibular ${ }^{4,28}$ e à menor inclinação mandibular e ângulo goníaco menos obtuso ${ }^{4,8}$. A espessura do músculo masseter acompanha a largura da maxila e este se torna mais espesso com a idade em indivíduos jovens ${ }^{47}$. Os resultados obtidos na correlação com a espessura da porção anterior do temporal demonstraram que este músculo não parece relacionar-se às variáveis DB e DI. Não há dados para comparações na literatura em crianças de pouca idade, tampouco trabalhos que tivessem avaliado e relacionado a espessura dos músculos mastigatórios às dimensões faciais nesta faixa etária.

Os resultados obtidos neste estudo permitiram concluir que a influência das características funcionais sobre as estruturas que compõem o sistema 
estomatognático pode ser observada já em crianças de pouca idade. Isto evidencia a importância da atenção precoce multidisciplinar às maloclusões, para que planos de tratamento efetivos possam ser estabelecidos, objetivando o adequado desenvolvimento morfológico associado ao desenvolvimento funcional, permitindo que o sistema estomatognático desenvolva-se e desempenhe sua função harmonicamente.

\section{CONCLUSÃO}

Os resultados obtidos na amostra estudada constataram que: a porção anterior do músculo temporal no repouso apresentou maior espessura no lado cruzado no grupo mista-cruzada ( $\mathrm{p}=0,05)$; a espessura do mús- culo masseter não apresentou diferença significativa entre os lados em todos os grupos; as variáveis corporais peso e altura não apresentaram correlação com a espessura dos músculos mastigatórios e crianças com faces mais longas apresentaram menor espessura do músculo masseter. Tais resultados mostraram que a influência das características funcionais sobre as estruturas que compõem o sistema estomatognático pode ser observada em crianças de pouca idade.

\section{AGRADECIMENTOS}

Agradecimentos a Dra. Nadia Lunardi (MS, Departamento de Odontologia Infantil - Área de Ortodontia da FOP - UNICAMP).

\begin{abstract}
Purpose: to evaluate the ultrasonographic thickness of masseter and anterior portion of the temporalis muscle and facial dimensions in children with normal occlusion and unilateral crossbite, in deciduous and early mixed dentition. Methods: the sample comprised 49 children of both genders, divided into four groups: deciduous-normal occlusion, deciduous-crossbite, mixed-normal occlusion and mixedcrossbite. Muscle thickness was compared between the right and left sides (normal occlusion) and normal and crossbite-side (crossbite) (Student's "t" test) and related to facial dimensions and body variables (Pearson's and Spearman's correlation test). Facial dimensions were determined by measuring standardized frontal photographs: anterior facial height (AFH), bizygomatic (FWB) and intergonial width (FWI), and $\mathrm{AFH} / \mathrm{FWB}$ and $\mathrm{AFH} / \mathrm{FWI}$ ratios. Results: the results showed that the anterior temporalis muscle at rest was significantly thicker in the crossbite side in the mixed-crossbite group $(p=0.05)$. Masseter thickness did not show significant differences between the sides in all groups. Weight and height did not correlate with masticatory muscle thickness; but FWB and FWI showed being positive, and $\mathrm{AFH} / \mathrm{FWB}$ and $\mathrm{AFH} / \mathrm{FWI}$ ratios showed having negative correlation with masseter thickness, i.e. children with a long-face pattern showed smaller muscle thickness. Conclusion: in the sample studied, the influence of functional characteristics on craniofacial structures was observed in young children by masticatory muscles ultrasonography and facial dimensions exam.
\end{abstract}

KEYWORDS: Ultrasonography; Masseter Muscle; Temporal Muscle; Malocclusion; Face

\section{REFERÊNCIAS}

1. Kiliaridis S, Kalebo P. Masseter muscle thickness measured by ultrasonography and its relation to facial morphology. J Dent Res. 1991; 70(9):1262-5.

2. Close PJ, Stokes MJ, L'Estrange PR, Rowell J. Ultrasonography of masseter muscle size in normal young adults. J Oral Rehabil. 1995; 22(2):129-34.

3. Raadsheer MC, Kiliaridis S, Van Eijden TM, Van Ginkel FC, Prahl-Andersen B. Masseter muscle thickness in growing individuals and its relation to facial morphology. Arch Oral Biol. 1996; 41(4):323-32.

4. Benington PC, Gardener JE, Hunt NP. Masseter muscle volume measured using ultrasonography and its relationship with facial morphology. Eur J Orthod. 1999;21(6):659-70.
5. Raadsheer MC, van Eijden TM, van Ginkel FC, Prahl-Andersen B. Contribution of jaw muscle size and craniofacial morphology to human bite force magnitude. J Dent Res. 1999; 78(1):31-42.

6.Kiliaridis S, Katsaros C, Raadsheer MC, Mahboubi $\mathrm{PH}$. Bilateral masseter muscle thickness in growing individuals with unilateral crossbite [abstract 2831]. J Dent Res. 2000; 79:497.

7.Bertram S, Brandlmaier I, Rudisch A, Bodner G, Emshoff R. Cross-sectional characteristics of the masseter muscle: an ultrasonographic study. Int J Oral Maxillofac Surg. 2003; 32(1):64-8. 8. Bakke $M$, Tuxen A, Vilmann $P$, Jensen BR, Vilmann A, Toft M. Ultrasound image of human masseter muscle related to bite force, electromyography, facial morphology, and occlusal factors. Scand J Dent Res. 
1992; 100(3):164-71.

9. Kiliaridis S, Kjellberg H, Wenneberg B, Engstrom C. The relationship between maximal bite force, bite force endurance, and facial morphology during growth. A cross-sectional study. Acta Odontol Scand. 1993; 51(5):323-31.

10. Silva Filho OG, Ferrari Júnior FM, Aiello CA, Zopone N. Correção da mordida cruzada posterior nas dentaduras decídua e mista. Rev APCD. 2000; 54(2):142-7. 11. Kiliaridis S. Masticatory muscle influence on craniofacial growth. Acta Odontol Scand. 1995; 53(3):196-202.

12. Varrela J, Alanen P. Prevention and early treatment in orthodontics: a perspective. J Dent Res. 1995; 74(8):1436-8.

13. Troelstrup B, Moller E. Electromyography of the temporalis and masseter muscles in children with unilateral cross-bite. Scand J Dent Res. 1970; 78(5):425-30.

14. Ingervall $B$, Thilander B. Activity of temporal and masseter muscles in children with a lateral forced bite. Angle Orthod. 1975; 45(4):249-58.

15. Alarcón JA, Martin C, Palma JC. Effect of unilateral posterior crossbite on the electromyographic activity of human masticatory muscles. Am $\mathrm{J}$ Orthod Dentofacial Orthop. 2000; 118(3):328-34.

16. Gavião MB, Raymundo VG, Sobrinho LC. Masticatory efficiency in children with primary dentition. Pediatr Dent. 2001;23(6):499-505.

17. Kutin G, Hawes RR. Posterior cross-bites in the deciduous and mixed dentitions. Am J Orthod. 1969; 56(5):491-504.

18. Sonnesen L, Bakke M, Solow B. Bite force in preorthodontic children with unilateral crossbite. Eur J Orthod. 2001; 23(6):741-9.

19. Rentes AM, Gaviao MB, Amaral JR. Bite force determination in children with primary dentition. J Oral Rehabil. 2002; 29(12):1174-80.

20. Saitoh I, Hayasaki H, Iwase Y, Nakata M. Improvement in jaw motion following treatment of unilateral crossbite in a child with primary dentition: a case report. Cranio. 2002; 20(2):129-34.

21. Foster TD, Hamilton MC. Occlusion in the primary dentition: study of children at 2 and one-half to 3 years of life. Br Dent J. 1969; 126(2):76-9.

22. Bonjardim LR, Gaviao MB, Carmagnani FG, Pereira LJ, Castelo PM. Signs and symptoms of temporomandibular joint dysfunction in children with primary dentition. J Clin Pediatr Dent. 2003; 28(1):53-8. 23. Landgraf ME, Vedovello Filho M, Junqueira JLC, Valdrighi HC, Vedovello SAS. Análise facial, elemento chave do diagnóstico ortodôntico contemporâneo. Ortod. 2002; 35(2):147-60.

24. Moreira RWF, Gabrielli MFR, Passeri LA, Moraes $M$. Análise facial e cefalométrica de mulheres com harmonia facial. Ortod. 2002; 35(1):51-7.

25. van der Bilt A. Human oral function: a review. Braz
J Oral Sci. 2002; 1(1):7-18.

26. Ingervall B, Minder C. Correlation between maximum bite force and facial morphology in children. Angle Orthod. 1997; 67(6):415-22.

27. Sonnesen L, Bakke M. Molar bite force in relation to occlusion, craniofacial dimensions, and head posture in pre-orthodontic children. Eur J Orthod. 2005; 27(1):58-63.

28. Kubota M, Nakano H, Sanjo I, Satoh K, Sanjo T, Kamegai T, et al. Maxillofacial morphology and masseter muscle thickness in adults. Eur J Orthod. 1998; 20(5):535-42.

29. Rasheed SA, Prabhu NT, Munshi AK. Electromyographic and ultrasonographic observations of masseter and anterior temporalis muscles in children. J Clin Pediatr Dent. 1996; 20(2):127-32.

30. Myers DR, Barenie JT, Bell RA, Williamson EH. Condylar position in children with functional posterior crossbites: before and after crossbite correction. Pediatr Dent. 1980; 2(3):190-4.

31. Langberg BJ, Arai K, Miner RM. Transverse skeletal and dental asymmetry in adults with unilateral lingual posterior crossbite. Am J Orthod Dentofacial Orthop. 2005; 127(1):6-15.

32. Planas P. Reabilitação neuroclusal. 2. ed. Rio de Janeiro: Medsi; 1997.

33. Oyen OJ. A função mastigatória e o crescimento e desenvolvimento facial. In: Enlow DH. Crescimento facial. 3. ed. São Paulo: Artes Médicas; 1993. p. 272-90.

34. Tsarapatsani P, Tullberg M, Lindner A, Huggare J. Long-term follow-up of early treatment of unilateral forced posterior cross-bite: orofacial status. Acta Odontol Scand. 1999; 57(2):97-104.

35. Salioni MA, Pellizoni SE, Guimaraes AS, Juliano Y, Alonso LG. Functional unilateral posterior crossbite effects on mastication movements using axiography. Angle Orthod. 2005; 75(3):362-7.

36. van Spronsen PH, Weijs WA, Valk J, PrahlAndersen B, van Ginkel FC. Comparison of jawmuscle bite-force cross-sections obtained by means of magnetic resonance imaging and high-resolution CT scanning. J Dent Res. 1989; 68(12):1765-70.

37. Yawaka Y, Hironaka S, Akiyama A, Matzuduka I, Takasaki C, Oguchi H. Changes in occlusal contact area and average bite pressure during treatment of anterior crossbite in primary dentition. J Clin Pediatr Dent. 2003; 28(1):75-9.

38. Proffit WR, Fields HW. Occlusal forces in normal- and long-face children. J Dent Res. 1983; 62(5):571-4.

39. Colombo VL, Moro A, Reche R, Verona J, Costa GCA. Facial frontal analysis in rest position and maximun smile in standardized photographs (in Portuguese). Rev Dent Press Ortod Ortop Facial. 2004; 9:47-58.

40. Katz CRT, Rosenblatt A, Gondim PPC. Nonnutritive 
sucking habits in Brazilian children: effects on deciduous dentition and relationship with facial morphology. Am J Orthod Dentofacial Orthop. 2004; 126(1):53-7.

41. García-Morales P, Buschang PH, Throckmorton GS, English JD. Maximum bite force, muscle efficiency and mechanical advantage in children with vertical growth patterns. Eur J Orthod. 2003; 25(3):265-72.

42. Tuxen A, Bakke M, Pinholt EM. Comparative data from young men and women on masseter muscle fibres, function and facial morphology. Arch Oral Biol. 1999; 44(6):509-18.

43. Rowlerson A, Raoul G, Daniel Y, Close J, Maurage CA, Ferri J, et al. Fiber-type differences in masseter muscle associated with different facial morphologies. Am J Orthod Dentofacial Orthop. 2005; 127(1):37-46.

44. Fields HW, Proffit WR, Nixon WL, Phillips C, Stanek E. Facial pattern differences in long-faced children and adults. Am J Orthod. 1984; 85(3):217-23.

45. Kovero O, Hurmerinta K, Zepa I, Huggare J, Nissinen M, Kononen M. Maximal bite force and its associations with spinal posture and craniofacial morphology in young adults. Acta Odontol Scand. 2002; 60(6):365-9.

46. Sassouni V. A classification of skeletal facial types. Am J Orthod. 1969; 55(2):109-23.

47. Kiliaridis S, Georgiakaki I, Katsaros C. Masseter muscle thickness and maxillary dental arch width. Eur J Orthod. 2003; 25(3):259-63.

RECEBIDO EM: 19/04/06

ACEITO EM: 05/11/06

Endereço para correspondência:

Rua Cotoxó, 637 ap.142

São Paulo - SP

CEP: $05021-000$

Tel: (11) 38011858 / 82024569 / 33994854

E-mail:paulacastelo@fop.unicamp.bre pcastelo@yahoo.com 